\title{
Genome-wide association study and candidate gene analysis of rice cadmium accumulation in grain in a diverse rice collection
}

Junliang Zhao ${ }^{1,2 \dagger}$, Wu Yang ${ }^{1,2+}$, Shaohong Zhang ${ }^{1,2}$, Tifeng Yang ${ }^{1,2}$, Qin Liu ${ }^{1,2}$, Jingfang Dong ${ }^{1,2}$, Hua Fu $u^{1,2}$, Xingxue Mao ${ }^{1,2}$ and Bin Liu ${ }^{1,2^{*}}$ (D)

\begin{abstract}
Background: Cadmium (Cd) accumulation in rice followed by transfer to the food chain causes severe health problems in humans. Breeding of low $\mathrm{Cd}$ accumulation varieties is one of the most economical ways to solve the problem. However, information on the identity of rice germplasm with low $\mathrm{Cd}$ accumulation is limited, particularly in indica, and the genetic basis of $\mathrm{Cd}$ accumulation in rice is not well understood.
\end{abstract}

Results: Screening of 312 diverse rice accessions revealed that the grain $\mathrm{Cd}$ concentrations of these rice accessions ranged from 0.12 to $1.23 \mathrm{mg} / \mathrm{kg}$, with 24 accessions less than $0.20 \mathrm{mg} / \mathrm{kg}$. Three of the 24 accessions belong to indica. Japonica accumulated significantly less $\mathrm{Cd}$ than indica $(p<0.001)$, while tropical japonica accumulated significantly less $\mathrm{Cd}$ than temperate japonica $(p<0.01)$. GWAS in all accessions identified 14 QTLs for $\mathrm{Cd}$ accumulation, with 7 identified in indica and 7 identified in japonica subpopulations. No common QTL was identified between indica and japonica. The previously identified genes (OsHMA3, OsNRAMP1, and OsNRAMP5) from japonica were colocalized with QTLs identified in japonica instead of indica. Expression analysis of OsNRAMP2, the candidate gene of the novel QTL ( $q C d 3-2)$ identified in the present study, demonstrated that OsNRAMP2 was mainly induced in the shoots of high $\mathrm{Cd}$ accumulation accessions after $\mathrm{Cd}$ treatment. Four amino acid differences were found in the open reading frame of OsNRAMP2 between high and low Cd accumulation accessions. The allele from low $\mathrm{Cd}$ accumulation accessions significantly increased the $\mathrm{Cd}$ sensitivity and accumulation in yeast. Subcellular localization analysis demonstrated OsNRAMP2 expressed in the tonoplast of rice protoplast.

Conclusion: The results suggest that grain $\mathrm{Cd}$ concentrations are significantly different among subgroups, with $\mathrm{Cd}$ concentrations decreasing from indica to temperate japonica to tropical japonica. However, considerable variations exist within subgroups. The fact that no common QTL was identified between indica and japonica implies that there is a different genetic basis for determining Cd accumulation between indica and japonica, or that some QTLs for $\mathrm{Cd}$ accumulation in rice are subspecies-specific. Through further integrated analysis, it is speculated that OsNRAMP2 could be a novel functional gene associated with $\mathrm{Cd}$ accumulation in rice.

Keywords: Rice (Oryza sativa L.), Cadmium accumulation, Quantitative trait locus (QTL), Genome-wide association study (GWAS)

\footnotetext{
*Correspondence: Ibgz1009@163.com

†Junliang Zhao and Wu Yang contributed equally to this work.

${ }^{1}$ Guangdong Key Laboratory of New Technology in Rice Breeding,

Guangzhou 510640, China

${ }^{2}$ Rice Research Institute, Guangdong Academy of Agricultural Sciences,

Guangzhou 510640, China
} 


\section{Background}

Cadmium (Cd) is one of the most mobile and toxic heavy metals. Due to rapid industrialization and environmental pollution, $\mathrm{Cd}$ pollution has become a major problem in paddy fields. It has been reported that approximately 20 million hectares of cultivated lands in China, or $20 \%$ of the total, were contaminated by $\mathrm{Cd}$ (Hu et al. 2016). Cd can be readily absorbed by the roots of crops and transferred to aboveground organs, then enter the human body through the food chain (Li et al. 2017). Rice is a staple food for nearly half of the world's population, and compared to other cereal crops, rice tends to accumulate more $\mathrm{Cd}(\mathrm{Hu}$ et al. 2016). A recent survey showed that $10 \%$ of rice grain with $\mathrm{Cd}$ exceeded the allowable concentration of $0.2 \mathrm{mg} / \mathrm{kg}$ as stipulated by the National Food Hygiene Standard of China (Xie et al. 2017). As rice is the primary source of dietary Cd intake (Clemens and Ma 2016; Uraguchi and Fujiwara 2013), $\mathrm{Cd}$ contamination in rice is becoming a severe public health problem.

Several techniques have been proposed to minimize $\mathrm{Cd}$ contamination in rice, such as phytoremediation and soil washing. Murakami et al. (2009) demonstrated that phytoextraction with the indica rice Chokoukoku, grown for 2 years without irrigation after drainage, removed $883 \mathrm{~g}$ $\mathrm{Cd} \mathrm{ha}{ }^{-1}$, reducing the total soil Cd content by $38 \%$. Makino et al. (2008) tested the feasibility of extracting Cd from the soil with iron, manganese, and zinc salts. Their results revealed that manganese, zinc, and iron salts extracted $4-41 \%, 8-44 \%$, and $24-66 \%$ of the total $\mathrm{Cd}$, respectively. Although these techniques are effective, they are time-consuming, expensive, or impractical in some situations. Many studies have revealed that $\mathrm{Cd}$ accumulation in rice varied considerably among cultivars and suggested that the breeding of low $\mathrm{Cd}$-accumulating rice cultivars is a feasible strategy (Arao and Ae 2003; Cao et al. 2014; Yu et al. 2006). To date, breeding and use of rice cultivars with low $\mathrm{Cd}$ concentration in grain has been considered a promising solution to reducing $\mathrm{Cd}$ contamination in rice.

Obtaining rice germplasm with low $\mathrm{Cd}$ accumulation and identifying the genes responsible for $\mathrm{Cd}$ intake and transport are the prerequisites for the effective breeding of low-Cd-accumulation rice. In the past decade, much effort has been dedicated to the identification of rice germplasm with low Cd accumulation and of QTLs/genes controlling Cd accumulation (Ishikawa et al. 2005; Miyadate et al. 2011; Shimo et al. 2011). Ishikawa et al. (2005) conducted the first QTL analysis of $\mathrm{Cd}$ accumulation in rice, using 39 chromosome segment substitution lines derived from "Kasalath" and "Koshihikari". They identified QTLs controlling the Cd concentration on chromosomes 3, 6, and 8 in brown rice. Since then, many QTLs for Cd accumulation in rice have been identified and mapped (Ueno et al. 2009; Xue et al. 2009; Norton et al. 2010; Ishikawa et al.
2010; Abe et al. 2013). Several genes have been cloned, and their functions have been confirmed regarding intake and transport of $\mathrm{Cd}$ in rice (Clemens and Ma 2016; Uraguchi and Fujiwara 2013). The $\mathrm{P}_{1 \mathrm{~B}}$-type Heavy Metal ATPase 3 (OsHMA3) is the first cloned gene responsible for $\mathrm{Cd}$ accumulation in rice (Miyadate et al. 2011; Ueno et al. 2010). OsHMA3 is expressed in the tonoplast of root cells, and limits translocation of $\mathrm{Cd}$ from the roots to the aboveground tissues by selectively sequestrating $\mathrm{Cd}$ into the root vacuoles (Miyadate et al. 2011; Ueno et al. 2010). Some indica and temperate japonica cultivars contain the non-functional allele of OsHMA3, which is related to higher $\mathrm{Cd}$ accumulation in these cultivars (Ueno et al. 2010; Yan et al. 2016). OsHMA2 is another HMA family gene confirmed to be responsible for $\mathrm{Cd}$ accumulation in rice. Studies on OsHMA2 suggest that it plays a role in $\mathrm{Cd}$ loading to the xylem and participates in root-to-shoot translocation of Cd (Satoh-Nagasawa et al. 2012; Takahashi et al. 2012). Another study by Yamaji et.al (2013) found that OsHMA2 also plays an important role in preferential distribution of $\mathrm{Cd}$ through the phloem to the developing tissues. Additionally, OsNRAMP1 and OsNRAMP5, belonging to the natural resistance-associated macrophage protein (NRAMP) family, were also identified as Cd transporters in rice (Sasaki et al. 2012; Takahashi et al. 2011). OsNRAMP1 participates in cellular Cd uptake and Cd transport within plants, and the higher expression of OsNRAMP1 in the roots could lead to an increase in Cd accumulation in the shoots (Takahashi et al. 2011). OsNRAMP5 is a plasma membrane-localized protein polarly localized at the distal side of both exodermis and endodermis cells. Functional analysis revealed that OsNRAMP5 is responsible for the transport of $\mathrm{Mn}$ and $\mathrm{Cd}$ from the external solution to root cells (Ishikawa et al. 2012; Sasaki et al. 2012). Despite significant progress being made in the identification of rice germplasm and associated QTLs/ genes with low $\mathrm{Cd}$ accumulation, identification of both the indica germplasm with low accumulation and functional genes associated with low $\mathrm{Cd}$ accumulation from indica are limited.

Fortunately, rice germplasm is very rich, providing a good condition in searching for rice cultivars with low $\mathrm{Cd}$ accumulation. Genome-wide association study (GWAS) has emerged as a powerful approach for identifying the genes underlying complex traits at an unprecedented rate (Huang et al. 2010). This approach was first applied to the genetic analysis of human diseases (Hirschhorn and Daly 2005), and then used extensively for the genetic dissection of complex traits in plants (Li et al. 2016; Weng et al. 2011; Yang et al. 2014). With the rapid development in next-generation sequencing technology, GWAS with high-density molecular markers and more diverse resources is possible. Recently, McCouch et al. (2016) established an open-access resources for 
GWAS in rice, which included a collection consisting of 1568 diverse inbred rice varieties and their genotypes determined by 700,000 single-nucleotide polymorphisms (SNPs), providing a good resource for the genetic dissection of complex traits in rice through GWAS.

To identify rice germplasms with low $\mathrm{Cd}$ accumulation and the responsible genes, 312 diverse rice accessions, selected from the 1568 rice accessions (McCouch et al. 2016), were subjected to evaluation of $\mathrm{Cd}$ accumulation; GWAS was performed to map QTLs associated with low $\mathrm{Cd}$ accumulation in the present study. Twenty-four rice accessions were identified, including three indica varieties, with grain $\mathrm{Cd}$ accumulation less than $0.2 \mathrm{mg} / \mathrm{kg}$. In total, 14,7 , and 7 QTLs for low Cd accumulation in grain were identified by GWAS, using all 312 rice accessions, in the indica subpopulation, and in the japonica subpopulation, respectively. The previously identified functional genes, including OsHMA3, OsNRAMP1, OsNRAMP5, and OsLCD, co-localized with the QTLs identified in the present study, suggesting the reliability of the GWAS results. Through differential expression analysis, genomic sequence analysis, yeast expression experiments, and subcellular localization analysis, Os03g0208500, a member of the rice NRAMP gene family, was identified as the possible functional gene of a novel QTL ( $q C d 3-2)$ identified in the present study. The results provide a good basis for cloning of the gene and molecular breeding for low $\mathrm{Cd}$ accumulation in rice.

\section{Results}

\section{Phylogenetic analysis of $\mathbf{3 1 2}$ rice accessions}

A total of 312 rice accessions, which are from 53 countries worldwide, were selected from 1568 rice accessions (McCouch et al. 2016) according to their diversity of genotypes, origins, and subpopulations. Phylogenetic tree (Fig. S1) of the selected 312 rice accessions was constructed using SNP data from the previous study (McCouch et al. 2016). The phylogenetic analysis showed that 312 rice accessions could be divided into two subpopulations, namely indica population and japonica population, according to their genetic similarities. The indica population consists of 213 rice accessions, while the $j a-$ ponica population consists of 99 rice accessions. The $j a-$ ponica population can be further divided into two groups (temperate japonica group and tropical japonica group) according to the phylogenetic analysis and information provided by the previous study (McCouch et al. 2016).

\section{Variations in cd accumulation in grain among 312 rice accessions}

Substantial variation and normal distribution in grain $\mathrm{Cd}$ accumulation were observed in 312 rice accessions (Fig. 1A). The indica cultivar "BOL ZO" from Korea Republic had the lowest $\mathrm{Cd}$ accumulation in grain $(0.12 \mathrm{mg} /$ $\mathrm{kg}$ ), while the indica cultivar "Jariyu" from India had the highest $\mathrm{Cd}$ accumulation in grain $(1.23 \mathrm{mg} / \mathrm{kg})$. In total, 24 rice accessions had $\mathrm{Cd}$ accumulation in grain lower than $0.20 \mathrm{mg} / \mathrm{kg}$ (Table 1). Three out of those 24 rice accessions belong to indica and the others belong to tropical japon$i c a$. The comparisons in grain $\mathrm{Cd}$ accumulation of subspecies and subgroups revealed that the mean grain $\mathrm{Cd}$ accumulation of indica was significantly higher than that of japonica $(p<0.001)$ (Fig. 1B), while the mean grain $\mathrm{Cd}$ accumulation of temperate japonica was markedly higher than that of tropical japonica $(p<0.01)$ (Fig. 1C).

\section{Mapping of QTLs for cd accumulation in grain by GWAS}

Based on the criteria of having less than 15\% missing data and minor allele frequency (MAF) larger than $5 \%$ in the selected population, 183,884 SNPs were selected for GWAS from the SNP dataset in the present study (McCouch et al. 2016). The QQ plot is shown in Fig. 2A. According to the LD decay rate of 312 rice accessions, a significant $\mathrm{LD}$ decay was observed after $200 \mathrm{~kb}$ (Fig. 2B). Therefore, a region was considered as one QTL where it had more than two SNPs with $P<0.0001$ within a 200-kb genomic window. The Manhattan plot of the GWAS results is shown in Fig. 2C. In total, 14 QTLs with 49 SNPs were significantly associated with grain $\mathrm{Cd}$ accumulation in the 312 rice accessions (Table 2). These QTLs (designate as $q C d$ hereafter) distributed on chromosomes 1, 2, 3, 4, 7, 8 and 11, with detailed information of the 14 QTLs listed in Table 2 of this report. It is noted that three QTLs identified in the present study co-localized with the four previously cloned genes associated with $\mathrm{Cd}$ accumulation in rice (OsLCD, OsHMA3, OsNRAMP1, OsNRAMP5) (Miyadate et al. 2011; Sasaki et al. 2012; Shimo et al. 2011; Takahashi et al. 2011) (Fig. 2 D and 2E) (Table 2).

GWAS was further conducted using the indica and japonica subpopulations of the 312 rice accessions and compared with all the identified QTLs (Fig. 3). Since fewer SNPs were used for GWAS in indica and japonica subpopulations according to the criteria of MAF $>0.05$, the $p$-value was reduced for selecting significant SNPs to $p<0.001$. In total, 7 and 7 QTLs were identified in the indica and japonica subpopulations, respectively (Table 2). Comparisons of the QTLs identified in different populations indicated that $q C d 1, q C d 2-1, q C d 2-2, q C d 4$ and $q C d 8-1$, which were identified in the whole population, overlapped with $q C d$ ind-1, $q C d$ _ind-2-1, $q C d \_i n d-2-2, q C d \_$ind-4 and $q C d \_$_ind-8, which were identified in the indica subpopulation. The $q C d 2-3, q C d 3-2, q C d 7-1, q C d 7-2$ identified in the whole population overlapped with $q C d \_j a p-2, q C d$ jap-3, $q C d$ jap-7-1 and $q C d$ jap-7-2 from the japonica subpopulation. The three cloned genes functioning in rice $\mathrm{Cd}$ accumulation (OsHMA3, OsNRAMP1, OsNRAMP5) were also located within the interval of $q C d \_j a p-7-1$ and $q C d \_j a p-7-2$, respectively (Table 2 ). Interestingly, no common QTL was detected between the indica and japonica subpopulations (Fig. 3, Table 2). 


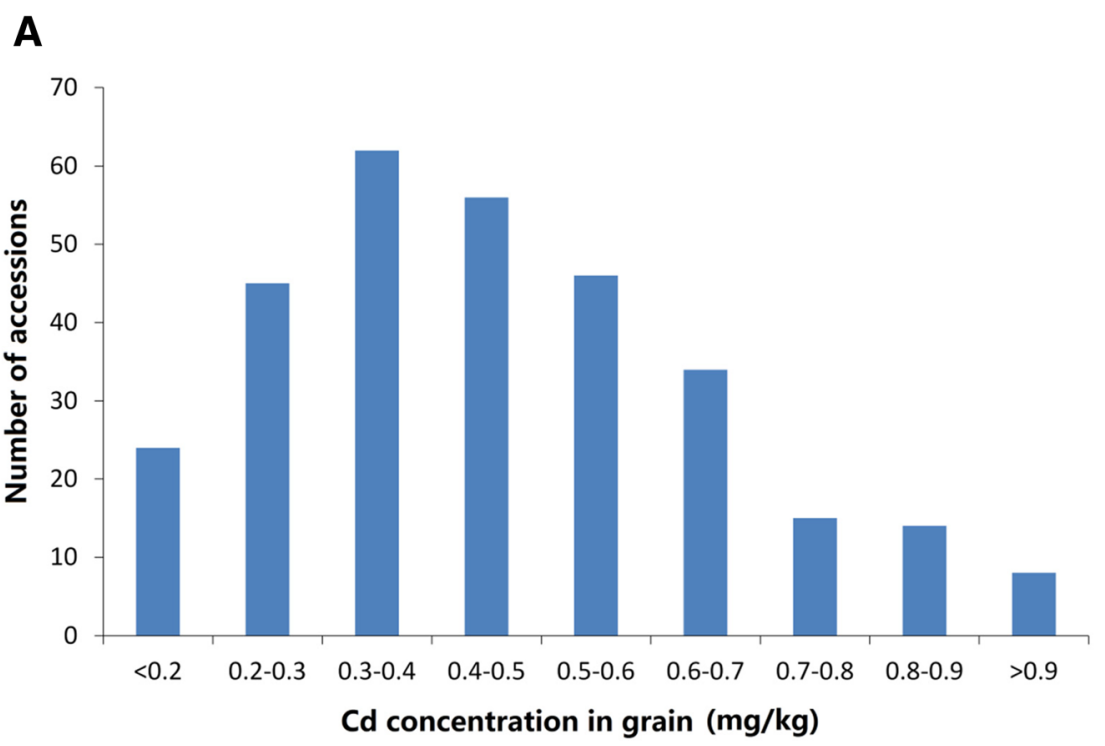

B

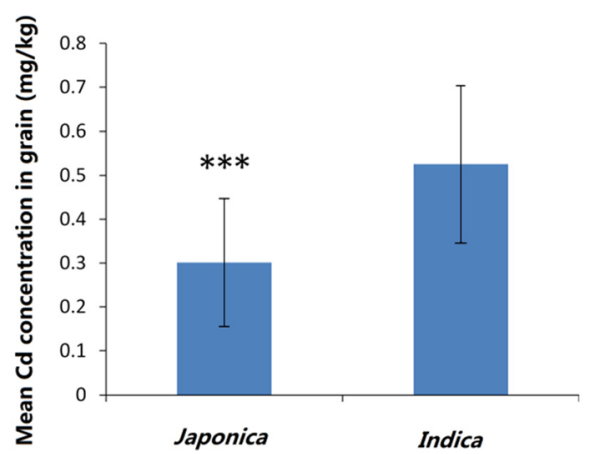

C

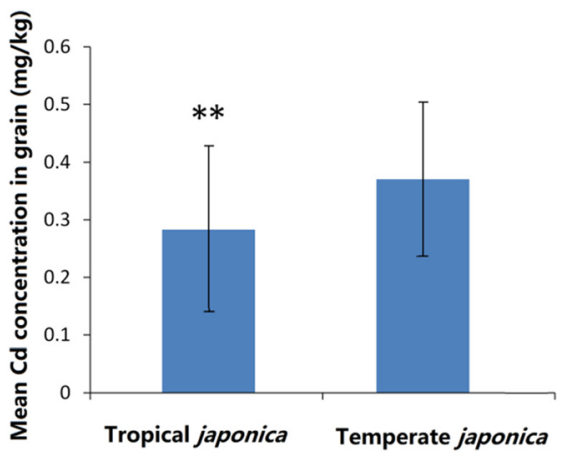

Fig. 1 Distribution of $\mathrm{Cd}$ concentration in grain in 312 rice accessions and comparisons of $\mathrm{Cd}$ concentration in grain between subspecies and between subgroups. a, Distribution of $\mathrm{Cd}$ concentration in grain of 312 rice accessions. $\mathbf{b}$, Comparison of $\mathrm{Cd}$ concentration in grain between indica and japonica species. c, Comparison of Cd concentration in grain between Temperate and Tropical japonica. ${ }^{* *}$ and ${ }^{* *}$ represent significant difference at $p<0.001$, and $p<0.01$ in $t$-test

\section{Identification of candidate genes related to cd accumulation in grain}

To identify candidate genes related to $\mathrm{Cd}$ accumulation in grain, annotations of the genes were analyzed within the identified QTLs (200 kb up and down stream of the most significant SNP of the QTL) based on the Rice Annotation Project (RAP) (Kawahara et al. 2013). Based on its annotation, a potential functional gene (Os03g0208500) belongs to the rice NRAMP gene family and is located $14 \mathrm{~kb}$ downstream from the most significant SNP in $q C d 3-2$ (Fig. $4 \mathrm{~A}$ ). The gene symbol synonym of Os03g0208500 in RAP is OsNRAMP2. Since other two members, OsNRAMP1 and OsNRAMP5, have been reported to function in $\mathrm{Cd}$ transportation in rice (Sasaki et al. 2012; Takahashi et al. 2011), it was reasoned that OsNRAMP2 might be the functional gene of $q C d 3-2$ associated with grain $\mathrm{Cd}$ accumulation in rice.
To confirm the function of OsNRAMP2 in Cd accumulation in rice, the gene structure of OsNRAMP2 and its expression patterns in both high and low $\mathrm{Cd}$ accumulation rice accessions were analyzed. The haplotypes associated with high and low $\mathrm{Cd}$ accumulation were determined by analyzing SNPs in the $q C d 3-2$ region and grain $C d$ accumulation of the tested rice accessions. In total, 15 rice accessions with low $\mathrm{Cd}$ accumulation phenotype and low $\mathrm{Cd}$ accumulation haplotype, and 15 accessions with the contrary phenotype and haplotype were selected. The genomic sequences of OsNRAMP2 in the selected 30 rice accessions were amplified by PCR and individually sequenced. Sequence analysis revealed four SNPs and one Indel in the coding sequence (CDS) of OsNRAMP2 among the 30 rice accessions (Fig. 4B). Among these differences, there were one Indel and three SNPs between the 15 high $\mathrm{Cd}$ accumulation accessions and 
Table 1 The rice accessions with Cd concentration lower than $0.2 \mathrm{mg} / \mathrm{kg}$ in grain

\begin{tabular}{|c|c|c|c|}
\hline Accession name & Subpopulation & Origin & $\begin{array}{l}\text { Cd concentration } \\
\text { in grain }(\mathrm{mg} / \mathrm{kg})\end{array}$ \\
\hline$\overline{B O L ~ Z O}$ & Indica & Korea Rep & 0.12 \\
\hline IAC 164 & Tropical Japonica & Brazil & 0.13 \\
\hline CHERIVIRUPPU & Indica & India & 0.14 \\
\hline LEVANTE HOMEM & Tropical Japonica & Brazil & 0.15 \\
\hline PACHOLINHA & Tropical Japonica & Brazil & 0.15 \\
\hline ARAGUAIA & Tropical Japonica & Brazil & 0.15 \\
\hline KWADWO AMOA & Tropical Japonica & Ghana & 0.15 \\
\hline GBANTE & Tropical Japonica & Ivory Coast & 0.16 \\
\hline MUT IAC 25-44-807 & Tropical Japonica & Guyana & 0.16 \\
\hline TOANG & Tropical Japonica & Indonesia & 0.16 \\
\hline KETAN MERAH & Tropical Japonica & Indonesia & 0.16 \\
\hline PATO DE GALLINAZO & Tropical Japonica & Australia & 0.16 \\
\hline BOTRA FOTSY & Tropical Japonica & Madagascar & 0.16 \\
\hline VARY MAINTY & Tropical Japonica & Madagascar & 0.16 \\
\hline VARY SOMOTRA SIHANAKA & Tropical Japonica & Madagascar & 0.17 \\
\hline MANGAVAVA FOTSILANSTSIKA & Tropical Japonica & Madagascar & 0.17 \\
\hline BAKAW & Tropical Japonica & Philippines & 0.18 \\
\hline HONDURAS & Tropical Japonica & Spain & 0.18 \\
\hline IRAT 364 & Tropical Japonica & Nicaragua & 0.18 \\
\hline ARC 18294 & Indica & India & 0.19 \\
\hline RXAR RGUE: & Tropical Japonica & United States & 0.19 \\
\hline WAB 56-125 & Tropical Japonica & Ivory Coast & 0.19 \\
\hline $1-52-6$ & Tropical Japonica & Brazil & 0.19 \\
\hline BOMALASANG & Tropical Japonica & Philippines & 0.19 \\
\hline
\end{tabular}

the 15 low $\mathrm{Cd}$ accumulation accessions except for the rice accession 194 from this study. The sequences of allele from low $\mathrm{Cd}$ accumulation accessions (designated as OsNRAMP2-L) are exactly the same as that from Nipponbare. The allele from high $\mathrm{Cd}$ accumulation accessions (designated as $\mathrm{OsN}$ $R A M P 2-H)$ has two amino acid deletions in the 24th and 25th position, as well as an amino acid mutation at the 235th position, with Glu being substituted by Asp, and the 508th amino acid Arg being substituted by Gln. None of these amino acids are within the putative conserved domains previously identified (Cellier et al. 1995; Mani and Sankaranarayanan 2018). However, according to the alignment results with all rice NRAMP genes (Mani and Sankaranarayanan 2018), the Arg in the 508th position is a conserved amino acid across all 7 rice NRAMP genes. This transition may have some functional implications.

To further validate the function of OsNRAMP2 in rice $\mathrm{Cd}$ accumulation, the expression pattern of OsNRAMP2 in shoot and roots was investigated. The four rice accessions with high $\mathrm{Cd}$ accumulation, as well as four accessions with low $\mathrm{Cd}$ accumulation, were selected from the 30 rice accessions above. Results demonstrated that the expression levels of OSNRAMP2 did not significantly change in the roots of any of the eight rice accessions after Cd treatment, compared to their respective controls $(p>0.05)$ (Fig. 4C). However, the expression levels of OsNRAMP2 in the shoots of the high $\mathrm{Cd}$ accumulation rice accessions increased after $\mathrm{Cd}$ treatment, while no significant change was observed in low $\mathrm{Cd}$ accumulation rice accessions (Fig. 4D).

To investigate $\mathrm{Cd}$ transport function of the two alleles of OsNRAMP2, expression vectors (pYES2) containing OsNRAMP2-H and OsNRAMP2-L were used to transform the yeast INVSc1 strain and $\Delta y c f 1$ mutant strain. The $\Delta y c f 1$ mutant strain is sensitive to $\mathrm{Cd}$ treatment. There was no difference in $\mathrm{Cd}$ sensitivity between yeast strains expressing vector control or different alleles of OsNRAMP2 in the presence of glucose in both the INVSc1 strain and $\Delta y c f 1$ mutant strain (Fig. 5A, B). In the presence of galactose, which induced gene expression, significant increase in $\mathrm{Cd}$ sensitivity was observed in yeast strain $\Delta y c f 1$ under $5 \mu \mathrm{M}$ of $\mathrm{Cd}$ treatment, and in yeast strain INVSc1 under $10 \mu \mathrm{M}$ of $\mathrm{Cd}$ treatment, when expressing OsNRAMP2-L. However, OsNRAMP2-H only slightly enhanced yeast $\mathrm{Cd}$ sensitivity compared to the 
A

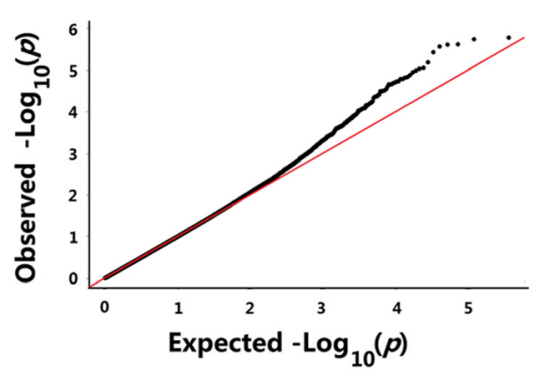

C
B

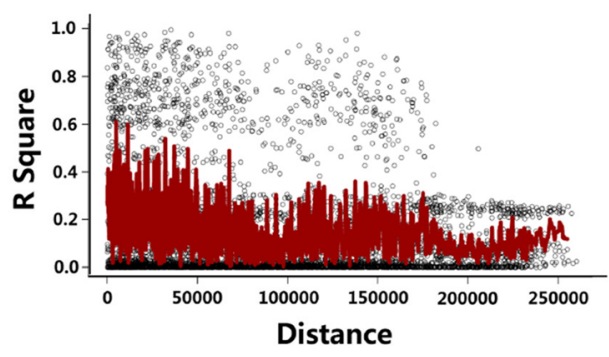

Distance

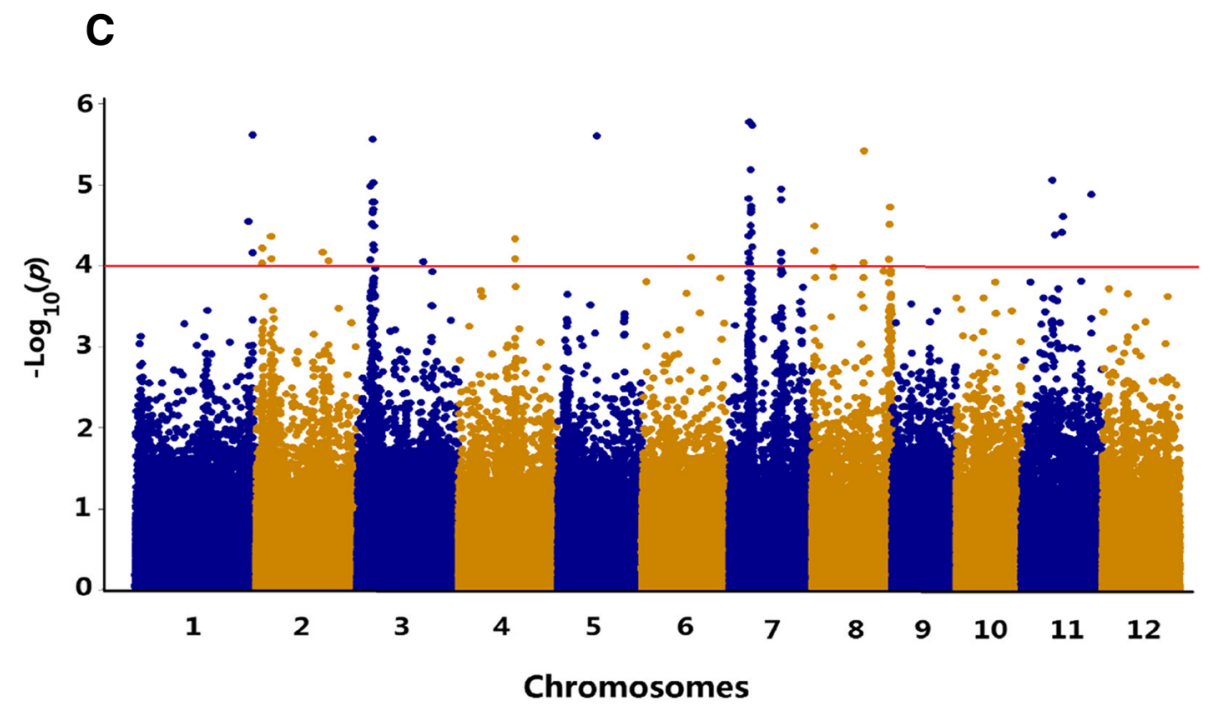

D

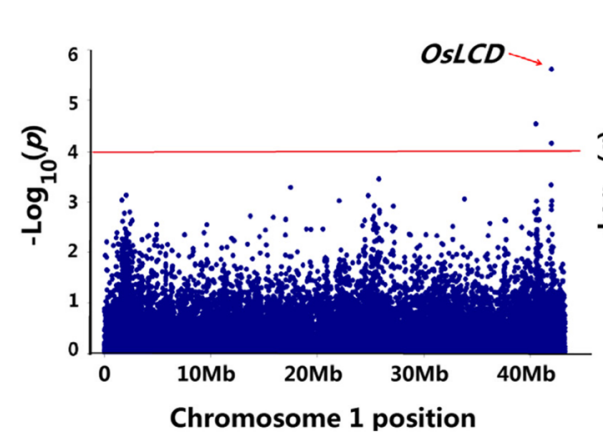

E

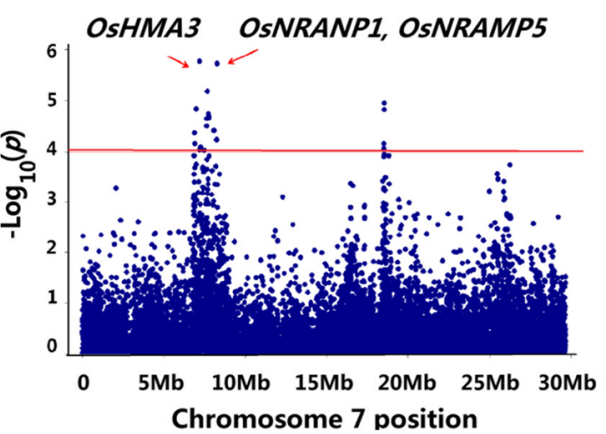

Fig. 2 GWAS of grain Cd accumulation using the 312 rice accessions. a, QQ-plot for GWAS of Cd concentration in grain. b, LD decay of the whole population. $\mathbf{c}$, Manhattan plots of GWAS of grain Cd accumulation in 12 chromosomes. $\mathbf{d}$, Colocalization of OsLCD with qCd-1. e, Co-localization of OsHMA3, OsNRAMP1 and OsNRAMP5 with qCd3-1 and qCd3-2

empty vector (Fig. 5C, D). Further analysis of the Cd accumulation in $\triangle y c f 1$ strain expressing different alleles demonstrated that yeast expressing OsNRAMP2- $L$ accumulated more $\mathrm{Cd}$ than those expressing OsNRAMP2-H or the empty vector (Fig. 5E). These results indicate that the OsNRAMP2-L has Cd transport function in yeast, but the $\mathrm{Cd}$ transport function of OsNRAMP2-H is reduced due to the change in amino acid sequence.
Subcellular localization of OsNRAMP2 protein was further investigated in rice protoplasts. Enhanced green fluorescent protein (EGFP) or OsNRAMP2-L/OsNRAMP2-H fused with EGFP at the C-terminal (designate as OsNRAMP2-L::EGFP and OsNRAMP2-H::EGFP) were transiently expressed in protoplasts from rice seedlings (Zhang et al. 2011). Under confocal microscopy, the green fluorescent signal of both the OsNRAMP2-L::EGFP and 
Table 2 QTL associated with Cd accumulation identified by GWAS using different populations

\begin{tabular}{|c|c|c|c|c|c|}
\hline$\overline{\text { QTLS }}$ & Chromosome & Linked SNP position $^{\text {a }}$ & $P$-value & Phenotype contribution (\%) & Candidate gene \\
\hline \multicolumn{6}{|c|}{ Composite population } \\
\hline$q C d 1$ & 1 & $41,982,531$ & $2.43 \mathrm{E}-06$ & 4.66 & OsLCD \\
\hline$q C d 2-1$ & 2 & $2,060,980$ & $6.01 \mathrm{E}-05$ & 2.40 & \\
\hline$a C d 2-2$ & 2 & $5,523,027$ & $4.38 \mathrm{E}-05$ & 3.47 & \\
\hline $9 C d 2-3$ & 2 & $23,732,572$ & $6.88 \mathrm{E}-05$ & 2.71 & \\
\hline$q C d 3-1$ & 3 & $4,848,498$ & $1.04 \mathrm{E}-05$ & 4.06 & \\
\hline $9 \mathrm{Cd} 3-2$ & 3 & $5,635,117$ & $2.78 \mathrm{E}-06$ & 4.61 & \\
\hline$q C d 4$ & 4 & $20,074,216$ & 4.67E-05 & 2.53 & \\
\hline$q C d 7-1$ & 7 & $7,186,204$ & 1.69E-06 & 4.82 & OsHMA3 \\
\hline$q C d 7-2$ & 7 & $8,263,482$ & $1.86 \mathrm{E}-06$ & 4.78 & $\begin{array}{l}\text { OsNRAMP1, } \\
\text { OsNRAMP5 }\end{array}$ \\
\hline $9 C d 7-3$ & 7 & $18,553,958$ & $1.14 \mathrm{E}-05$ & 4.02 & \\
\hline $9 C d 8-1$ & 8 & 902,085 & $3.25 \mathrm{E}-05$ & 3.01 & \\
\hline $9 \mathrm{Cd} 8-2$ & 8 & $18,365,933$ & $3.82 \mathrm{E}-06$ & 4.48 & \\
\hline $9 C d 8-3$ & 8 & $27,463,194$ & $3.12 \mathrm{E}-05$ & 2.60 & \\
\hline qCd11 & 11 & $14,891,373$ & $2.48 \mathrm{E}-05$ & & \\
\hline \multicolumn{6}{|l|}{ Indica } \\
\hline aCd_ind_1 & 1 & $41,982,531$ & 8.89E-05 & 6.85 & OsLCD \\
\hline qCd_ind_2-1 & 2 & $2,060,980$ & 7.66E-04 & 5.00 & \\
\hline qCd_ind_2-2 & 2 & $4,986,931$ & $2.27 \mathrm{E}-04$ & 6.04 & \\
\hline qCd_ind_3 & 3 & $27,195,551$ & $3.06 \mathrm{E}-04$ & 5.78 & \\
\hline qCd_ind_4 & 4 & $20,368,258$ & $1.34 \mathrm{E}-04$ & 5.93 & \\
\hline qCd_ind_5 & 5 & $23,588,056$ & 4.53E-04 & 5.45 & \\
\hline qCd_ind_8 & 8 & 899,377 & $1.93 \mathrm{E}-05$ & 8.20 & \\
\hline \multicolumn{6}{|l|}{ Japonica } \\
\hline qCd_jap-2 & 2 & $23,666,135$ & $5.23 \mathrm{E}-05$ & 12.74 & \\
\hline qCd_jap-3 & 3 & $5,507,369$ & $3.81 \mathrm{E}-04$ & 9.63 & \\
\hline qCd_jap-7-1 & 7 & $7,451,789$ & $3.52 \mathrm{E}-04$ & 9.75 & OsHMA3 \\
\hline qCd_jap-7-2 & 7 & $8,467,983$ & $1.56 \mathrm{E}-04$ & 11.01 & $\begin{array}{l}\text { OsNRAMP1, } \\
\text { OsNRAMP5 }\end{array}$ \\
\hline qCd_jap-7-3 & 7 & $24,677,317$ & $3.71 \mathrm{E}-05$ & 13.29 & \\
\hline qCd_jap-7-4 & 7 & $27,037,840$ & $6.24 \mathrm{E}-04$ & 8.88 & \\
\hline qCd_jap-11 & 11 & $25,263,712$ & 4.09E-04 & 9.52 & \\
\hline
\end{tabular}

aposition of SNP is base on rice reference sequence MSU V 7.0. (Kawahara et al. 2013)

OsNRAMP2-H::EGFP was observed outside of the chloroplasts (the chloroplasts showed red signal) (Fig. 6). These results suggest that both proteins are localized to the tonoplast.

\section{Discussion}

Cd accumulation in grain is diverse in rice germplasm, and is significantly different among subspecies and subgroups

$\mathrm{Cd}$ is a highly toxic and mobile element. Consequently, it can easily enter the food chain and become detrimental to human and animal health. To minimize the influx of $\mathrm{Cd}$ into the human food chain through rice consumption, $\mathrm{Yu}$ et al. (2006) proposed the concept of pollution-safe cultivars (PSCs), i.e. cultivars whose edible parts accumulate a specific pollutant at a level low enough for safe consumption, even when grown in contaminated soil. Selecting or breeding low $\mathrm{Cd}$ accumulation cultivars is an attractive and economical method for minimizing the Cd contamination in rice, as the benefit will persist in the seed and reduces the need for other management techniques such as fertilization management or crop rotations. 

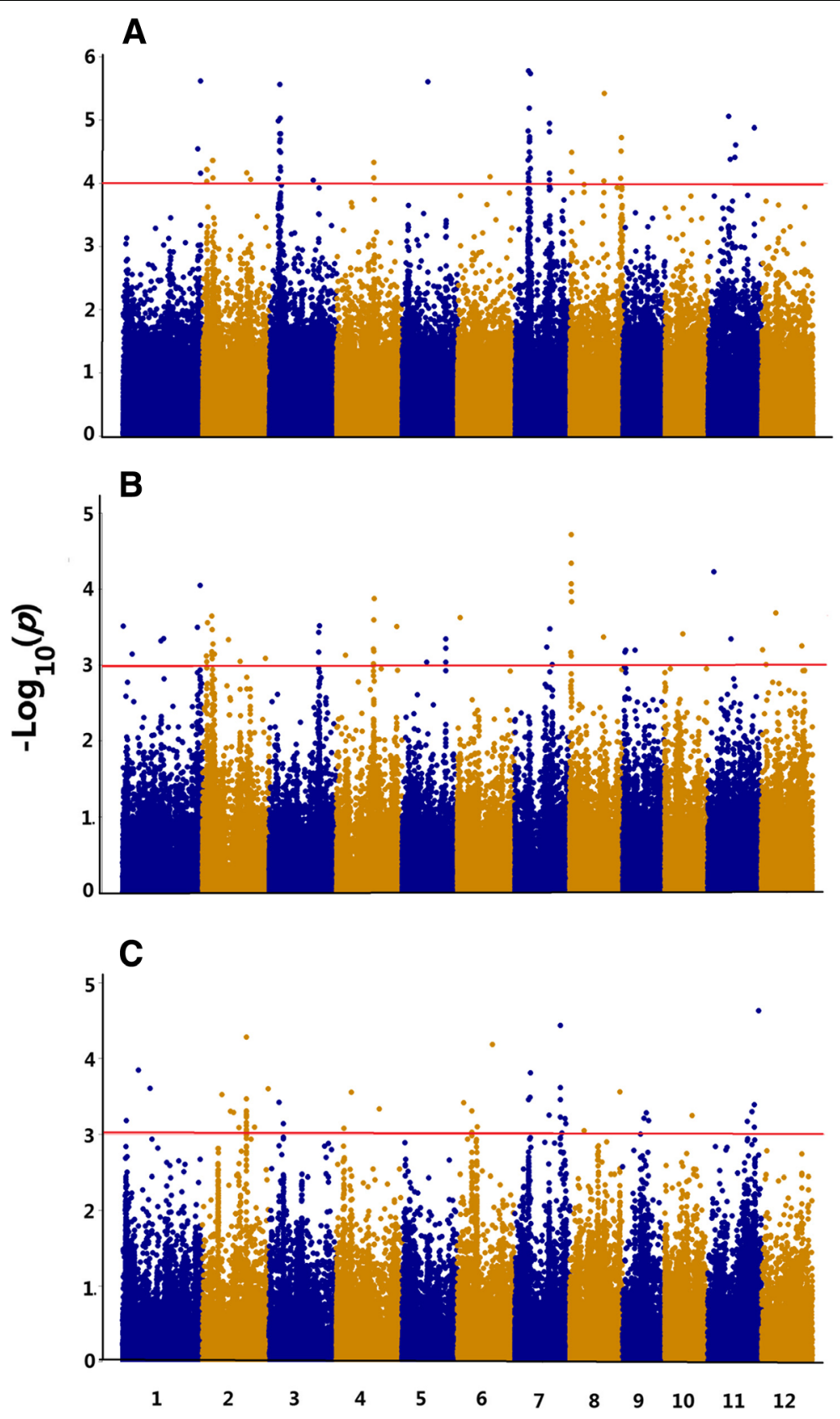

\section{Chromosomes}

Fig. 3 Manhattan plots of GWAS of grain Cd accumulation using different populations. a, Composite population. b, Indica subpopulation. c, Japonica subpopulation

To search for rice resources with low $\mathrm{Cd}$ accumulation for breeding, a diverse rice collection consisting of 312 rice accessions was subjected to evaluation of $\mathrm{Cd}$ accumulation. The results showed that $\mathrm{Cd}$ concentration in grains among the 312 rice accessions varied considerably, ranging from $0.12 \mathrm{mg} / \mathrm{kg}$ to $1.23 \mathrm{mg} / \mathrm{kg}$ when these rice accessions were grown in Cd-contaminated paddy fields (Fig. 1A). Similar to previous studies (Arao and Ae 2003, Liu et al. 2005; Pinson et al. 2015; Zhou et al. 2017), the results indicate that the mean grain $\mathrm{Cd}$ accumulation in japonica accessions is significantly lower than that in indica accessions $(p<0.001)$ (Fig. 1B). Furthermore, the mean grain Cd concentration in tropical japonica accessions is significantly lower than that in temperate japonica accessions (Fig. 1C). This result contradicts previous findings claiming that temperate japonica has lower $\mathrm{Cd}$ accumulation compared to tropical japonica (Yan 


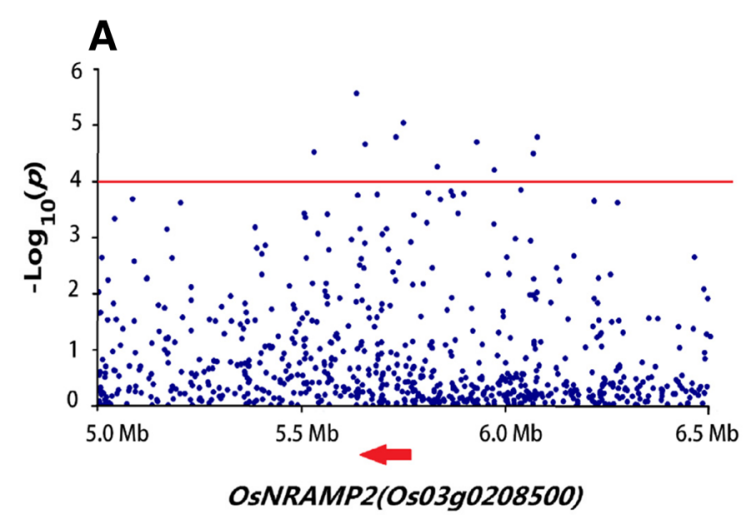

B
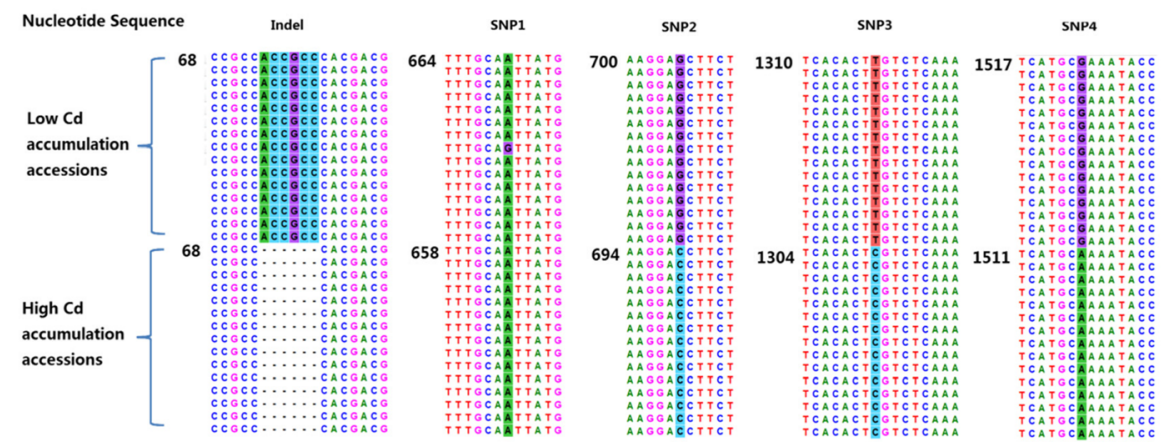

Protein Sequence
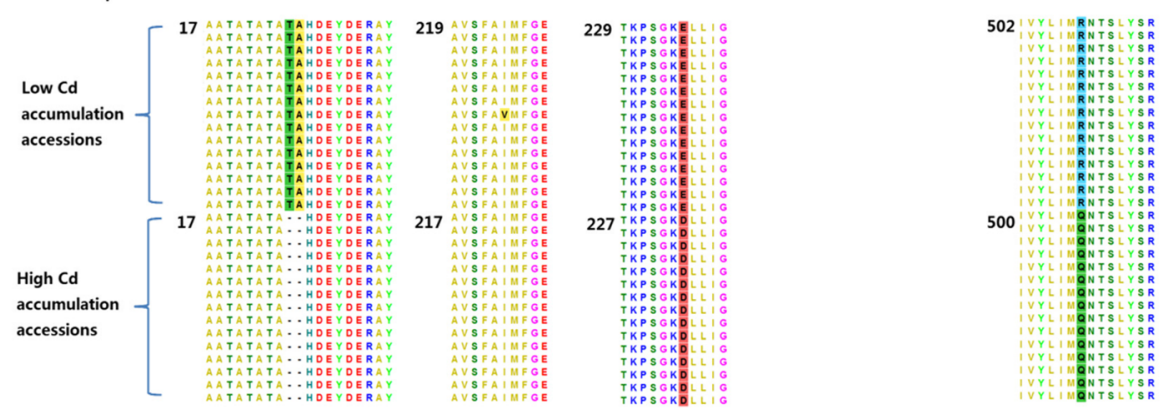

C

D
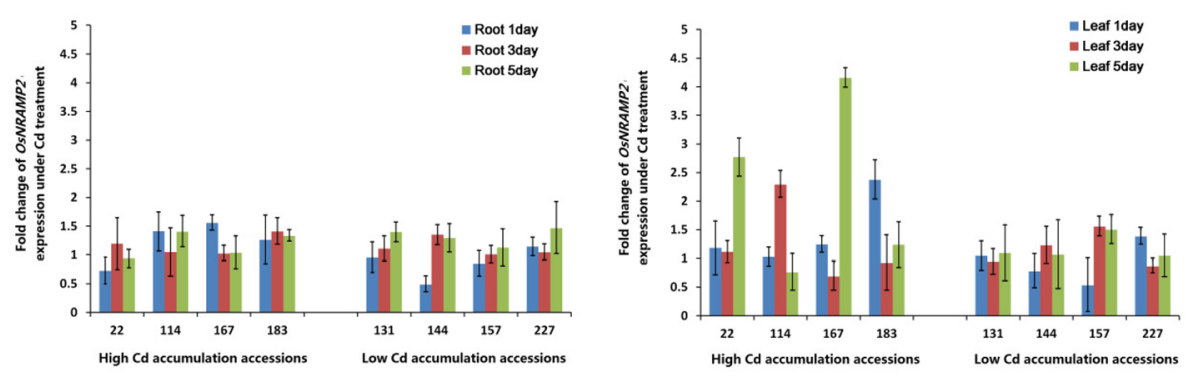

Fig. 4 (See legend on next page.) 
(See figure on previous page.)

Fig. 4 The location of OSNRAMP2 on chromosome 3 and sequence differences and expression analysis of OsNRAMP2. a, Colocalization of OsNRAMP2 with aCd3-2. The red arrow indicates the location and direction of OsNRAMP2. b, Sequence differences of OsNRAMP2 protein and the respective causal nucleotide differences between high and low $\mathrm{Cd}$ accumulation accessions. Sequence differences between high and low $\mathrm{Cd}$ accumulation rice accessions are indicated with different background colors. The numbers on the left of each local alignment indicate the position of the first nucleotide/amino acid for the alignment. c, Expression changes of OSNRAMP2 in roots after Cd treatment between high and low $\mathrm{Cd}$ accumulation rice accessions. $\mathbf{d}$, Expression changes of OsNRAMP2 in shoot after $\mathrm{Cd}$ treatment between high and low $\mathrm{Cd}$ accumulation rice accessions

et al. 2010). Although indica cultivars accumulate more $\mathrm{Cd}$, three indica accessions with grain Cd concentrations lower than $0.20 \mathrm{mg} / \mathrm{kg}$ were still identified. Interestingly, the indica "BOL ZO" from the Korean Republic had the lowest grain Cd concentration $(0.12 \mathrm{mg} / \mathrm{kg})$ among the 312 rice accessions (Table 1). Together, these results suggest that $\mathrm{Cd}$ accumulation in grain is diverse in rice germplasm. However, the $\mathrm{Cd}$ accumulation in grain is significant different among subgroups, with accumulation in indica > temperate japonica > tropical japonica. Regardless of subspecies or subgroups, it is possible to screen for rice germplasm with low $\mathrm{Cd}$ accumulation, but there is a higher likelihood of identifying them in tropical japonica.

\section{There is a different genetic basis for cd accumulation between indica and japonica rice}

In the present study, 14 QTLs related to Cd accumulation were identified through GWAS using a population consisting of 312 rice accessions. Chromosomal position comparisons reveal that the previously identified genes

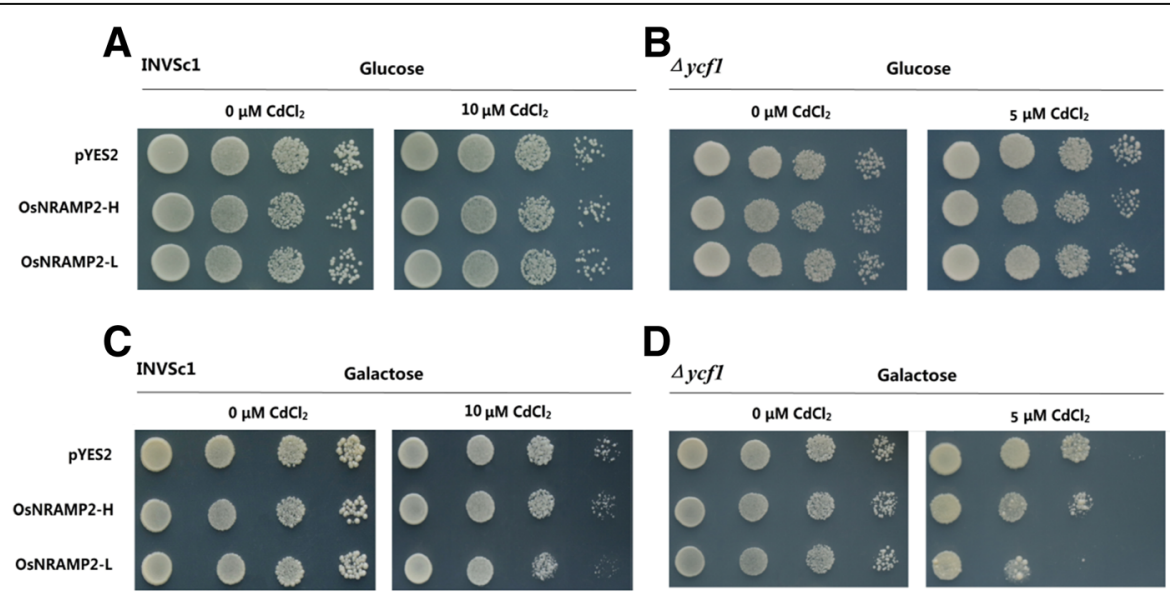

E

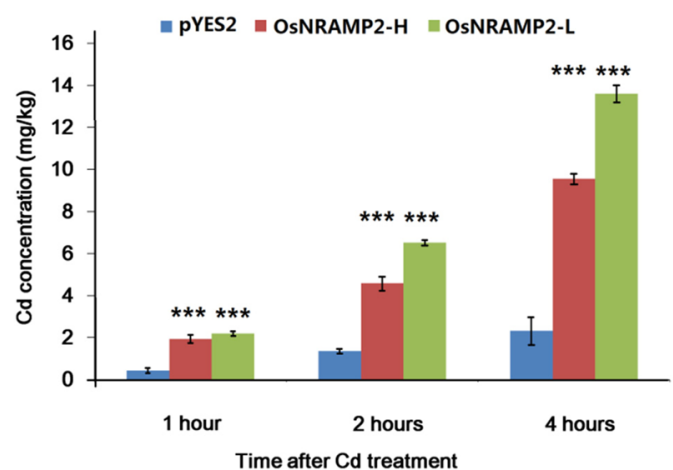

Fig. 5 Functional assay of OSNRAMP2 by heterologous expression in yeast. Yeast INVSc1 strain and $\triangle y c f 1$ mutant strain expressing the empty vector (pYES2), OsNRAMP2-H and OsNRAMP2-L were grown in SD-U medium containing concentrations of 0 and $5 \mu \mathrm{M} / 10 \mu \mathrm{M} C \mathrm{C}$ in the presence of glucose $(\mathbf{a}, \mathbf{b})$ to suppress the expression of transformed gene, or galactose $(\mathbf{c}, \mathbf{d})$ to induce the expression of transformed gene. E, $\mathrm{Cd}$ accumulation in $\triangle y c f 1$ strain expressing empty vector and OsNRAMP2-H, OsNRAMP2-L after exposure to $5 \mu M C d$ for 1,2 and $4 \mathrm{~h}$. Data are means \pm SD of three biological replicates. ${ }^{* *} p<0.001$ in $t$-test 


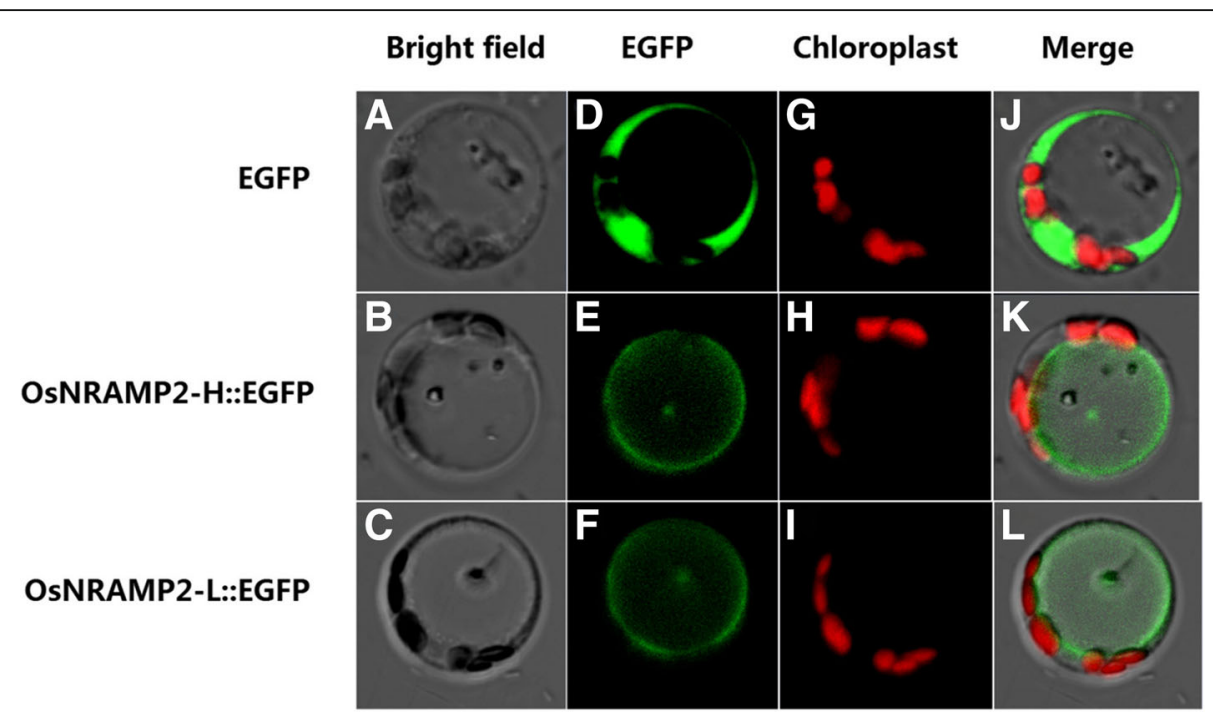

Fig. 6 Subcellular localization of OsNRAMP2. Images of bright field (a-c), EGFP-derived green fluorescence (d-f), chloroplast auto-fluorescence (g-i) and merge images (j-l) of protoplasts expressing EGFP, OsNRAMP2-H::EGFP and OsNRAMP2-L::EGFP

related to $C d$ transportation and accumulation, OsLCD, OsHMA3, OsNRAMP1 and OsNRAMP5, are located within the QTLs $q C d 1, q C d 7-1$ and $q C d 7-2$ identified in the present study, indicating the reliability of GWAS results in this study.

Comparison of QTLs identified by GWAS in indica and japonica subpopulations indicates that no common QTL. The three genes, OsHMA3, OsNRAMP1 and OsNRAMP5 cloned from japonica, were co-localized with $q C d$ _jap-7-1 and $q C d$ jap-7-2 only in the japonica subpopulation. It was reported that some indica cultivars and temperate japonica cultivars contained a loss-of-function allele of OsHMA3, in which results in weakened vacuolar sequestration of $\mathrm{Cd}$ in the roots and an enhanced $\mathrm{Cd}$ accumulation, while many japonica cultivars contained a functional allele of OsHMA3 (Ueno et al. 2010; Yan et al. 2016). Similarly, a significant difference was observed in the expression levels of OsNRAMP1 between the indica and japonica subspecies, which may also explain the difference in Cd accumulation between these subspecies (Zhou et al. 2017). Our findings, together with the results from previous studies, suggest that there may be a different genetic basis of $\mathrm{Cd}$ accumulation in grain between indica and japonica, and the genes/QTLs responsible for Cd transportation and accumulation may be subspecies-specific.

Since indica rice accumulates more $\mathrm{Cd}$ and the cloned genes, so far, are from japonica rice, there is an urgent need to identify the genes related to $\mathrm{Cd}$ accumulation in indica rice and understand their molecular mechanisms. In the present study, three indica accessions with very low Cd concentrations in grain (Table 1) and 7 QTLs associated with $\mathrm{Cd}$ accumulation in indica have been identified by GWAS (Table 2), providing a good basis for the identification of genes responsible for $\mathrm{Cd}$ accumulation and molecular breeding for low $\mathrm{Cd}$ accumulation in indica rice.

OsNRAMP2 could be a novel functional gene controlling grain cd accumulation in rice

Through bioinfomatic analysis of the QTLs identified in the present study, OsNRAMP2 in the $q C d 3-2$ region was identified. NRAMP is a family of integral membrane proteins identified in many species (Cellier et al. 1995). Previous studies demonstrated that many members of this family could function as metal transporters in yeast and plants (Supek et al. 1996; Thomine et al. 2000). Particularly, various members of this family had been identified as Cd transporters in plants (Clemens and Ma 2016). For example, AtNRAMP6 is a Cd transporter that functions inside the cell either by mobilizing cadmium from its storage compartment or by bringing cadmium to the cellular compartment where it is toxic (Cailliatte et al. 2009).

Altogether, seven NRAMP genes have been identified in rice, and five of them (OsNRAMP1, 3, 4, 5, and 6) have been functionally characterized (Mani and Sankaranarayanan 2018). OsNRAMP1 and OsNRAMP5 were identified as Cd transporters in rice. OsNRAMP5 is responsible for the transport of $\mathrm{Mn}$ and $\mathrm{Cd}$ from the external solution to root cells, but the function of the OsNRAMP1 Cd transporter is not fully understood (Clemens and Ma 2016; Takahashi et al. 2011). However, the function of OsNRAMP2 as a Cd transporter had not been reported previously.

OsNRAMP2 localizes within a $200 \mathrm{~kb}$ region of $q C d 3-2$ as identified in the present study. There were four amino acid differences in the gene between high and low $\mathrm{Cd}$ accumulation accessions. The heterologous assay in 
yeast (Fig. 5) showed that OsNRAMP2-L can increase $\mathrm{Cd}$ sensitivity and accumulation in yeast, while OsNRAMP2-H only slightly increased Cd sensitivity and accumulation in yeast, suggesting that OsNRAMP2 could function as a $\mathrm{Cd}$ transporter and OsNRAMP2-L is its functional form. Furthermore, the subcellular localization analysis demonstrated that both OsNRAMP2-L and OsNRAMP2-H are localized at the tonoplast (Fig. 6). Base on previous studies, OsNRAMP1 and OsNRAMP5 are localized to the plasma membrane and mediate metal uptake into the cytosol (Sasaki et al. 2012; Takahashi et al. 2011). In the present study, the OsNRAMP2 protein was localized to the tonoplast, suggesting that it might mediate vacuole-to-cytosol transport of $\mathrm{Cd}$ as like AtNRAMP3 and AtNRAMP4, which influence metal accumulation by mobilizing vacuolar metal pool to the cytosol (Lanquar et al. 2005; Thomine et al. 2000; Thomine, et al. 2003). Further physiological, cellular and molecular studies are underway to elucidate the actual function and mechanism of OsNRAMP2 in Cd transport in rice.

\section{Conclusion}

In the present study, $\mathrm{Cd}$ accumulation in grain from a diverse rice collection was screened, and QTLs related to rice $\mathrm{Cd}$ accumulation were identified through GWAS. The results suggest that the $\mathrm{Cd}$ accumulation in grain is significantly different among subgroups (indica $>$ temperate japonica > tropical japonica). Substantial variation also exists within subgroups. It is possible to screen for indica germplasm with low $\mathrm{Cd}$ accumulation as long as diverse indica accessions are investigated, but more success could be found in tropical japonica. The fact that no common QTL was identified between the indica group and japonica group in the present study imply that there may be a different genetic basis for $\mathrm{Cd}$ accumulation between indica and japonica, or that many QTLs for Cd accumulation in rice are subspecies-specific. Through an integrated analysis using GWAS, gene annotation, and functional analysis, it is speculated that OsNRAMP2 could be a functional $\mathrm{Cd}$ transporter, and is considered as a novel candidate functional gene associated with $\mathrm{Cd}$ accumulation in rice. This study provides new insights into the genetic basis of $\mathrm{Cd}$ accumulation, as well as a novel candidate functional gene associated with rice $\mathrm{Cd}$ accumulation. These results lay a strong foundation for gene cloning and molecular breeding for low $\mathrm{Cd}$ accumulation in rice.

\section{Methods}

\section{Materials}

A total of 312 rice accessions from 53 countries were selected according to the 1568 diverse rice accessions based on their 700,000 SNP genotypes and their origins (McCouch et al. 2016). Seeds of all 312 lines are provided by the International Rice Research Institute (IRRI).

\section{Field experiment and cd treatment}

The field experiments were conducted in 2016 in Renhua, Guangdong, China. The fields are rice-growing paddy fields belonging to light loam soils. Paddy water management, fertilizer applications, and crop protection followed the local farming practices. The 312 rice accessions were planted in a randomized complete design with three replicates. Ten plants per accession were planted in each replicate. The $\mathrm{Cd}$ concentration of the field is $1.4 \mathrm{mg} / \mathrm{kg}$ on average, which is higher than the second criteria level of the National Environmental Quality Standard for Soil (GB 15618-1995) (People's Republic of China). The concentrations of other heavy metals $(\mathrm{Pb}, \mathrm{Cr}, \mathrm{Hg}, \mathrm{As})$ are lower than the national standard level 1 , indicating that the soil is not polluted by these heavy metals. The $\mathrm{pH}$ value of the field was 5.5.

\section{Sampling and cd detection}

To determine the grain $\mathrm{Cd}$ concentrations of the 312 rice accessions, 8 plants in the middle of the 10 plants in each replicate were harvested. The grains of eight plants in each replicate were pooled and dried in oven at $70^{\circ} \mathrm{C}$ for $24 \mathrm{~h}$, then de-husked. The de-husked grains were grounded into powder and digested with an acid mixture of $\mathrm{HNO}_{3}-\mathrm{H}-$ $\mathrm{ClO}_{4}$. The $\mathrm{Cd}$ concentration was determined using a Inductively Coupled Plasma Optical Emission Spectrometry (ICP-OES, iCAP6000,Thermo Scientific, USA).

\section{GWAS, QTL delimitation and identification of candidate gene}

GAPIT version 2 was used for GWAS analysis (Tang et al. 2016). SNPs were selected for GWAS analysis from the $700 \mathrm{~K}$ assay of a previous study by the criteria of having less than $15 \%$ missing data and minor allele frequency (MAF) > 0.05 (McCouch et al. 2016). GWAS was conducted using the mix liner model with kinship matrix, and PC was set to 2 in GAPIT. Manhattan and QQ plots were produced using $\mathrm{R}$ package qqman.

Rice genome sequence version of MSU V7.0 was used as reference for analysis (Kawahara et al. 2013). After GWAS analysis, we follow the criteria of having one associated locus between any two significant SNPs within a $200 \mathrm{~kb}$ interval. After determining the QTL of each GWAS analysis, the candidate genes were searched from $200 \mathrm{~kb}$ upstream and downstream of the most significant SNP in each QTL.

\section{Sequencing of OsNRAMP2 in the rice accessions with low and high cd accumulation}

The low and high $\mathrm{Cd}$ accumulation rice accessions with 15 accessions each were selected according to the haplotype associated with $\mathrm{Cd}$ accumulation within $q C d 3-2$ and $\mathrm{Cd}$ accumulation in the grains of 312 rice accessions. Seedlings of selected rice accessions were used for DNA extraction by the DNeasy Plant Mini Kit (Qiagen, Germany). The DNA samples of the selected rice accessions were used as 
templates to amplify the full-length genomic sequence of OsNRAMP2 by KOD-FX polymerase (Toyobo, Japan) using the following primers, forward primer: acaaccactcctagagtccagaga, reverse primer: ggcgacatctcctgaagataacct. After PCR amplification, PCR products were sequenced by Sangon Biotech Co., Ltd. (Shanghai, China). Sequences of $O s N$ $R A M P 2$ from the 30 accessions were aligned and analyzed by MEGA 7.0 (Kumar et al. 2016).

\section{Determining the expression patterns of OsNRAMP2 in rice after cd treatment}

Four rice accessions with high $\mathrm{Cd}$ accumulation and four rice accessions with low $\mathrm{Cd}$ accumulation were selected from the 30 rice accessions mentioned above to analyze the expression patterns of OsNRAMP2 after $\mathrm{Cd}$ treatment. Seeds of selected rice accessions were germinated and grown hydroponically in half-strength Kimura B solution for 2 weeks, with $\mathrm{pH}$ adjusted to 5.6 for the duration. The solution was renewed every two days. Plants were grown in an RXZ-1000C growth chamber (Ningbo Jiangnan Instrument Factory, NingBo, China) under a light intensity of $359 \mu \mathrm{mol} \mathrm{m}^{-2} \mathrm{~s}^{-1}$ with a $12 \mathrm{~h}$ day length and a relative humidity of $80 \pm 5 \%$. The temperature was maintained at $25^{\circ} \mathrm{C}$. After two weeks of growing, plants were treated with $5 \mu \mathrm{M} \mathrm{CdCl} 2$ for various days. After $\mathrm{Cd}$ treatment for $1 \mathrm{~d}, 3 \mathrm{~d}$ and $5 \mathrm{~d}$, plants were separated into root and shoot, and RNA was extracted by TRIzol (Thermo Fisher, USA) according to the manufacturer's instructions. Rice plants without $\mathrm{Cd}$ treatment were used as control. All assays were conducted in three biological replicates. The RNA samples were reversely transcribed using PrimeScript Reverse Transcriptase (Takara, Japan). Gene expression was determined by qRT-PCR using Biorad CFX96 (Biorad, USA) and SYBR Premix Ex Taq (Takara, Japan).

\section{Functional analysis of OsNRAMP2 alleles in yeast}

Saccharomyces cerevisiae strain INVSc1 (Yan et al. 2016) and Cd-sensitive mutant strain $\Delta y c f 1$ (Ueno et al. 2010) were used for heterologous expression of OsNRAMP2- $H$ and OsNRAMP2-L. The ORF of OsNRAMP2-H and OsN$R A M P 2-L$ were amplified by KOD FX (Toyobo, Japan) from the cDNA of high and low $\mathrm{Cd}$ accumulation accessions as described above, using the following primers: forward primer, aaaagaattcaaaaaaatgtctatggcgtcgcgcgacctcg, reverse primer, aaaactcgagatcatgtgctctttgtcattgct. PCR products were cloned into expression vector pYES2 (Thermo Fisher, USA). Transformation and selection of empty vector and vectors with OsNRAMP2 alleles were conducted according to a previous study (Ueno et al. 2010). Positive clones were cultured in SD medium without uracil (SD-U) liquid media with $2 \%$ glucose to the early $\log$ phase. Five $\mu \mathrm{L}$ of the cell suspension with an initial OD value of 0.1 and three serial 1:10 dilutions were spotted on SD-U plates containing 0 and $10 \mu \mathrm{M} \mathrm{CdCl}_{2}$ for the INVSc1 strain, and 0 and $5 \mu \mathrm{M} \mathrm{CdCl}_{2}$ for the $\Delta y c f 1$ strain, in the presence of $2 \%$ glucose or galactose. The plates were incubated at $30^{\circ} \mathrm{C}$ for $3 \mathrm{~d}$ before the growth phenotypes were evaluated. $\mathrm{Cd}$ accumulation in yeast $\triangle y c f 1$ strains containing pYES2 empty vector and vectors expressing OsNRAMP2 alleles was determined according to a previous study (Yan et al. 2016). The transformed yeast strains were cultured in SD-U liquid media containing $2 \%$ glucose to the early log phase, then enriched by centrifugation and washed with sterile water three times. Cells were then adjusted to the same density (OD600 value 0.2 ) in $\mathrm{SD}-\mathrm{U}$ liquid media containing $2 \%$ galactose. After incubation for $3 \mathrm{~h}$ to induce protein expression, the media was amended with $5 \mu \mathrm{M} \mathrm{CdCl}$ and the cells were sampled after culturing for 1,2 and $4 \mathrm{~h}$. The sampled cells were washed with a cold $\left(4^{\circ} \mathrm{C}\right)$ EDTA solution $(10 \mu \mathrm{M}$, $\mathrm{pH}$ 5.0) twice, and then with deionized water twice, and freeze-dried. The cells were weighed, and Cd concentration was determined as described above.

\section{Subcellular localization of OsNRAMP2 protein}

The CDS sequences of OsNRAMP2-L and OsNRAMP2-H were amplified using primers as following: forward primer, aaaagaattcatggcgtcgcgcgacctcg, reverse primer, aaaagtcgactgtgctctttgtcattgctgag. The CDS sequences were inserted into the 35S::EGFP vector to produce the OsNRAMP2-L::EGFP and OsNRAMP2-H::EGFP fusion proteins. The fusion plasmids and the empty 35S::EGFP plasmid were transferred into rice protoplasts according to a previous study (Zhang et al. 2011). Laser confocal microscopy (Zeiss, LSM710, Germany) was used to detect fluorescence after incubation for $24 \mathrm{~h}$.

\section{Data analysis}

Phylogenetic tree of all 312 lines was constructed by MEGA 7.0 (Kumar et al. 2016) using SNP data mentioned above. A t-test was conducted using SAS (SAS Institute, 2000) to detect the differences in $\mathrm{Cd}$ accumulation in grain between indica rice and japonica rice, and between tropical japonica rice and temperate japonica rice.

\section{Additional files}

Additional file 1: Figure S1. Phylogenetic tree of the 312 rice accessions based on their genotypes determined by 700 K SNPs (DOCX 279 kb)

Additional file 2: Table S1. The Cd accumulation in grain of the 312 rice accession in the present study. (XLSX $34 \mathrm{~kb}$ )

\section{Abbreviations}

Cd: Cadmium; CDS: Coding sequence; EGFP: Enhanced green fluorescent protein; GWAS: Genome-wide association study; InDel: Insertion-deletion; MAF: Minor allele frequency; NRAMP: Natural resistance-associated macrophage protein; PSC: Pollution-safe cultivar; QTL: Quantitative trait loci; SNP: Single-nucleotide polymorphism 


\section{Acknowledgments}

The seeds of the 312 rice accessions in the present study were provided by International Rice Research Institute. This study was partially supported by Guangdong Provincial Scientific and Technological Plan (2015B020231002, 2015B020201001), Science and Technology Program of Guangzhou (201510010279), the Team Project of Guangdong Agricultural Department (2017LM2148) and the Special Fund of Central Government Guided Local Scientific Development.

\section{Funding}

This study was partially supported by Guangdong Provincial Scientific and Technological Plan (2015B020231002, 2015B020201001), Science and Technology Program of Guangzhou (201510010279), the Team Project of Guangdong Agricultural Department (2017LM2148) and the Special Fund of Central Government Guided Local Scientific Development.

\section{Availability of data and materials}

The detail $\mathrm{Cd}$ accumulation data of the 312 rice accessions is available in the additional file (Table S1). The $700 \mathrm{~K}$ SNP genotype data of the 312 rice accessions can be downloaded in McCouch et al. (2016)

\section{Authors' contributions}

$J L Z, B L$, and $S H Z$ conceived and designed the experiment. $J L Z, W Y, S H Z$ conducted the experiments, performed data analysis and wrote the manuscript. TF Y, W Y, SH Z, Q L, JF D, H F and XX M participated in material development, sample preparation and data analysis. $B L$ and $J L Z$ drafted proposals and corrected the manuscript. All authors read and approved the final manuscript.

\section{Authors' information}

B L, Ph D in Plant Molecular Genetics, Professor and Head of the Rice Molecular Breeding Lab, Rice Research Institute, Guangdong Academy of Agricultural Sciences, China;

$J$ Z, Ph D in Plant Molecular Biology, Associate Professor of the Rice Molecular Breeding Lab, Rice Research Institute, Guangdong Academy of Agricultural Sciences, China:

W Y, Ph D in Plant Molecular Biology, Assistant Professor of the Rice Molecular Breeding Lab, Rice Research Institute, Guangdong Academy of Agricultural Sciences, China;

SH Z, M.S. in Plant Physiology and Biochemistry, Professor of the Rice Molecular Breeding Lab, Rice.

Research Institute, Guangdong Academy of Agricultural Sciences, China; TF Yang, Ph D in Plant Genetics and Breeding, Associate Professor of the Rice Molecular Breeding Lab, Rice Research Institute, Guangdong Academy of Agricultural Sciences, China;

Q L, Ph D in Plant Molecular Biology, Associate Professor of the Rice Molecular Breeding Lab, Rice Research Institute, Guangdong Academy of Agricultural Sciences, China;

JF D, Ph D in Plant Molecular Biology, Assistant Professor of the Rice Molecular Breeding Lab, Rice Research Institute, Guangdong Academy of Agricultural Sciences, China;

H F, B.S in Agronomy, Assistant Professor of the Rice Molecular Breeding Lab, Rice Research Institute, Guangdong Academy of Agricultural Sciences, China; XX M, Ph D in Plant Molecular Biology, Associate Professor of the Rice Molecular Breeding Lab, Rice Research Institute, Guangdong Academy of Agricultural Sciences, China;

Ethics approval and consent to participate Not applicable.

\section{Consent for publication}

Not applicable.

\section{Competing interests}

The authors declare that they have no competing interests.

\section{Publisher's Note}

Springer Nature remains neutral with regard to jurisdictional claims in published maps and institutional affiliations.
Received: 9 April 2018 Accepted: 14 November 2018

Published online: 21 November 2018

\section{References}

Abe T, Nonoue Y, Ono N, Omoteno M, Kuramata M, Fukuoka S, Yamamoto T, Yano M, Ishikawa S (2013) Detection of QTLs to reduce cadmium content in rice grains using LAC23/Koshihikari chromosome segment substitution lines. Breed Sci 63:284-291

Arao T, Ae N (2003) Genotypic variations in cadmium levels of rice grain. Soil Sci Plant Nutr 49:473-479

Cailliatte R, Lapeyre B, Briat JF, Mari S, Curie C (2009) The NRAMP6 metal transporter contributes to cadmium toxicity. Biochem J 422:217-228

Cao F, Wang R, Cheng W, Zeng F, Mosaddek Ahmed I, Hu X, Zhang G, Wu F (2014) Genotypic and environmental variation in cadmium, chromium, lead and copper in rice and approaches for reducing the accumulation. Sci Total Environ 496:275-281

Cellier M, Prive G, Belouchi A, Kwan T, Rodrigues V, Chia W, Gros P (1995) Nramp defines a family of membrane proteins. Proc Natl Acad Sci U S A 92:1008910093

Clemens S, Ma JF (2016) Toxic heavy metal and metalloid accumulation in crop plants and foods. Annu Rev Plant Biol 67(1):489-512

Hirschhorn J, Daly M (2005) Genome-wide association studies for common diseases and complex traits. Nat Rev Genet 6:95-108

Hu Y, Cheng H, Tao S (2016) The challenges and solutions for cadmiumcontaminated rice in China: a critical review. Environ Int 92:515-532

Huang X, Wei X, Sang T, Zhao Q, Feng Q, Zhao Y, Li C, Zhu C, Lu T, Zhang Z, Li M, Fan D, Guo Y, Wang A, Wang L, Deng L, Li W, Lu y WQ, Liu K, Huang T, Zhou T, Jing Y, Li W, Lin Z, Buckler E, Qian Q, Zhang Q, Li J, Han B (2010) Genome-wide association studies of 14 agronomic traits in rice landraces. Nat Genet 42:961-967

Ishikawa S, Abe T, Kuramata M, Yamaguchi M, Ando T, Yamamoto T, Yano M (2010) A major quantitative trait locus for increasing cadmium-specific concentration in rice grain is located on the short arm of chromosome 7. J Exp Bot 61:923-934

Ishikawa S, Ae N, Yano M (2005) Chromosomal regions with quantitative trait loc controlling cadmium concentration in brown rice (Oryza sativa). New Phytol 168:345-350

Ishikawa S, Ishimaru Y, Igura M, Kuramata M, Abe T, Senoura T, Hase Y, Arao T, Nishizawa NK, Nakanishi H (2012) Ion-beam irradiation, gene identification, and marker-assisted breeding in the development of low-cadmium rice. Proc Natl Acad Sci U S A 109:19166-19171

Kawahara Y, Bastide M, Hamilton J, Kanamori H, McCombie W, Ouyang S, Schwartz D, Tanaka T, Wu J, Zhou S, Childs K, Davidson R, Lin H, QuesadaOcampo L, Vaillancourt B, Sakai H, Lee S, Kim J, Numa H, Itoh T, Buell C, Matsumoto T (2013) Improvement of the Oryza sativa Nipponbare reference genome using next generation sequence and optical map data. Rice 6:4

Kumar S, Stecher G, Tamura K (2016) MEGA7: molecular evolutionary genetics analysis version 7.0 for bigger datasets. Mol Biol Evol 33:1870-1874

Lanquar V, Lelièvre F, Bolte S, Hamès C, Alcon C, Neumann D, Vansuyt G, Curie C, Schröder A, Krämer U, Barbier-Brygoo H, Thomine S (2005) Mobilization of vacuolar iron by AtNRAMP3 and AtNRAMP4 is essential for seed germination on low iron. EMBO J 24:4041-4051

Li F, Chen B, Xu K, Gao G, Yan G, Qiao J, Li J, Li H, Li L, Xiao X, Zhang T, Nishio T, Wu X (2016) A genome-wide association study of plant height and primary branch number in rapeseed (Brassica napus). Plant Sci 242:169-177

Li K, Yu H, Li T, Chen G, Huang F (2017) Cadmium accumulation characteristics of low-cadmium rice (Oryza sativa L.) line and F1 hybrids grown in cadmiumcontaminated soils. Environ Sci and Pollut Res 24:17566-17576

Liu J, Zhu Q, Zhang Z, Xu J, Yang J, Wong M (2005) Variations in cadmium accumulation among rice cultivars and types and the selection of cultivars for reducing cadmium in the diet. J Sci Food Agric 85:147-153

Makino T, Takano H, Kamiya T, Itou T, Sekiya N, Inahara M, Sakurai Y (2008) Restoration of cadmium-contaminated paddy soils by washing with ferric chloride: cd extraction mechanism and bench-scale verification. Chemosphere 70:1035-1043

Mani A, Sankaranarayanan K (2018) In silico analysis of natural resistanceassociated macrophage protein (NRAMP) family of transporters in rice. Protein J 37:237-247

McCouch S, Wright M, Tung C, Maron L, McNally K, Fitzgerald M, Singh N, DeClerck G, Agosto-Perez F, Korniliev GA, Naredo MS, Harrington S, Shi Y, Branchini D, Kuser-Falcao P, Leung H, Ebana K, Yano M, Eizenga G, McClung 
A, Mezey J (2016) Open access resources for genome-wide association mapping in rice. Nat Commun 7:10532

Miyadate H, Adachi S, Hiraizumi A, Tezuka K, Nakazawa N, Kawamoto T, Katou K, Kodama I, Sakurai K, Takahashi H, Satoh-Nagasawa N, Watanabe A, Fujimura T, Akagi H (2011) OsHMA3, a P1B-type of ATPase affects root-to-shoot cadmium translocation in rice by mediating efflux into vacuoles. New Phytol 189:190-199

Murakami M, Nakagawa F, Ae N, Ito M, Arao T (2009) Phytoextraction by rice capable of accumulating $\mathrm{cd}$ at high levels: reduction of $\mathrm{cd}$ content of rice grain. Environ Sci Technol 43:5878-5883

Norton GJ, Deacon CM, Xiong L, Huang S, Meharg AA, Price AH (2010) Genetic mapping of the rice ionome in leaves and grain: identification of qtls for 17 elements including arsenic, cadmium, iron and selenium. Plant Soil 329:139-153

Pinson SRM, Tarpley L, Yan W, Yeater K, Lahner B, Yakubova E, Huang XY, Zhang HM, Guerinot ML, Salt DE (2015) Worldwide genetic diversity for mineral element concentrations in rice grain. Crop Sci 55:294-311

SAS Institute (2000) SAS/STAT user's guide, version 8.1. SAS Institute Inc, Cary, North Carolina,USA

Sasaki A, Yamaji N, Yokosho K, Ma J (2012) Nramp5 is a major transporter responsible for manganese and cadmium uptake in rice. Plant Cell 24:21552167

Satoh-Nagasawa N, Mori M, Nakazawa N, Kawamoto T, Nagato Y, Sakurai K, Takahashi H, Watanabe A, Akagi H (2012) Mutations in rice (Oryza sativa) heavy metal ATPase 2 (OsHMA2) restrict the translocation of zinc and cadmium. Plant Cell Physiol 53:213-224

Shimo H, Ishimaru Y, An G, Yamakawa T, Nakanishi H, Nishizawa N (2011) Low cadmium (LCD), a novel gene related to cadmium tolerance and accumulation in rice. J Exp Bot 62:5727-5734

Supek F, Supekova L, Nelson H, Nelson N (1996) A yeast manganese transporter related to the macrophage protein involved in conferring resistance to mycobacteria. Proc Natl Acad Sci U S A 93:5105-5110

Takahashi R, Ishimaru Y, Senoura T, Shimo H, Ishikawa S, Arao T, Nakanishi H, Nishizawa N (2011) The OsNRAMP1 iron transporter is involved in cd accumulation in rice. J Exp Bot 62:4843-4850

Takahashi R, Ishimaru Y, Shimo H, Ogo Y, Senoura T, Nishizawa N, Nakanishi H (2012) The OsHMA2 transporter is involved in root-to-shoot translocation of $\mathrm{Zn}$ and $\mathrm{cd}$ in rice. Plant Cell Environ 35:1948-1957

Tang Y, Liu X, Wang J, Li M, Wang Q, Tian F, Su Z, Pan Y, Liu D, Lipkai A, Buckler E, Zhang Z (2016) GAPIT version 2: an enhanced integrated tool for genomic association and prediction. The Plant Genome 9:2

Thomine S, Lelièvre F, Debarbieux E, Schroeder Jl, Barbier-Brygoo H (2003) AtNRAMP3, a multispecific vacuolar metal transporter involved in plant responses to iron deficiency. Plant J 34:685-695

Thomine S, Wang R, Ward JM, Crawford NM, Schroeder JI (2000) Cadmium and Iron transport by members of a plant metal transporter family in Arabidopsis with homology to Nramp genes. Proc Natl Acad Sci U S A 97:4991-4996

Ueno D, Kono I, Yokosho K, Ando T, Yano M, Ma JF (2009) A major quantitative trait locus controlling cadmium translocation in rice (Oryza sativa). New Phytol 182:644-653

Ueno D, Yamaji N, Kono I, Huang C, Ando T, Yano M, Ma J (2010) Gene limiting cadmium accumulation in rice. Proc Natl Acad Sci U S A 107:16500-16505

Uraguchi S, Fujiwara T (2013) Rice breaks ground for cadmium-free cereals. Curr Opin Plant Biol 16:328-334

Weng J, Xie C, Hao Z, Wang J, Liu C, Li M, Zhang D, Bai L, Zhang S, Li X (2011) Genome-wide association study identifies candidate genes that affect plant height in Chinese elite maize (Zea mays L.) inbred lines. PLoS One 6:e29229

Xie L, Tang S, Wei X, Shao G, Jiao G, Sheng Z, Luo J, Hu P (2017) The cadmium and lead content of the grain produced by leading Chinese rice cultivars. Food Chem 217:217-224

Xue D, Chen M, Zhang G (2009) Mapping of QTLs associated with cadmium tolerance and accumulation during seedling stage in rice (Oryza sativa, L.). Euphytica 165:587-596

Yamaji N, Xia J, Mitaniueno N, Yokosho K, Ma JF (2013) Preferential delivery of zinc to developing tissues in rice is mediated by P-type heavy metal atpase OsHMA2. Plant Physiol 162:927-939

Yan J, Wang P, Wang P, Yang M, Lian X, Tang Z, Huang C, Salt D, Zhao F (2016) A loss-of-function allele of OsHMA3 associated with high cadmium accumulation in shoots and grain of japonica rice cultivars. Plant Cell Environ 39:1941-1954

Yan Y, Choi D, Kim D, Lee B (2010) Genotypic variation of cadmium accumulation and distribution in rice. J Crop Sci Biotech 13:69-73
Yang W, Guo Z, Huang C, Duan L, Chen G, Jiang N, Fang W, Feng H, Xie W, Lian X, Wang G, Luo Q, Zhang Q, Liu Q, Xiong L (2014) Combining highthroughput phenotyping and genome-wide association studies to reveal natural genetic variation in rice. Nat Commun 5:5087

Yu H, Wang J, Fang W, Yuan J, Yang Z (2006) Cadmium accumulation in different rice cultivars and screening for pollution-safe cultivars of rice. Sci Total Environ 370:302-309

Zhang Y, Su J, Duan S, Ao Y, Dai J, Liu J, Wang P, Li Y, Liu B, Feng D, Wang J, Wang $H$ (2011) A highly efficient rice green tissue protoplast system for transient gene expression and studying light/chloroplast-related processes. Plant Methods 7:30

Zhou Q, Shao G, Zhang Y, Dong Q, Wang H, Cheng S, Cao L, Shen X (2017) The difference of cadmium accumulation between the indica and japonica subspecies and the mechanism of it. Plant Growth Regul 81:523-532

\section{Submit your manuscript to a SpringerOpen ${ }^{\circ}$ journal and benefit from:}

- Convenient online submission

- Rigorous peer review

- Open access: articles freely available online

- High visibility within the field

- Retaining the copyright to your article

Submit your next manuscript at $>$ springeropen.com 